\title{
Design of Embedded Hybrid Fuzzy-GA Control Strategy for Speed Control of DC Motor: A Servo Control Case Study
}

\author{
Rahul Malhotra \\ Research Scholar, \\ Punjab Technical University, \\ Jalandhar, Punjab, India
}

\author{
Narinder Singh \\ Ex-Professor, Thapar University, \\ Patiala, Punjab, India \\ Director, GGS Group \\ Talwandi Sabo ( $\mathrm{Pb}$.), India
}

\author{
Yaduvir Singh \\ Associate Professor, \\ Thapar University, \\ Patiala, Punjab, India
}

\begin{abstract}
In this paper, DC motor speed control models were mathematically extracted and implemented using artificial intelligence techniques. Due to multivariable and non-linear in nature, it is difficult to achieve the optimum speed of the motor. Conventional PI controller gives the maximum overshoot problem. To overcome the maximum overshoot, artificial intelligence techniques have been incorporated in the controller architecture. Fuzzy logic controlled model of DC motor is implemented, investigated and further optimised by the genetic algorithm for the optimal fuzzy rule base. Digital signal processor TMS320LF2407A is also tested to control the speed of servo motor. The transient time, steady state response and the performance criteria IAE and ITAE of the above controllers have been investigated. The result shows that the fuzzy logic- GA integrated approach has minimum overshoot, and minimum transient and steady state parameters, which shows the more effectiveness and efficiency of FLC-GA hybridisation than FLC and conventional PI model to control the speed of the motor.
\end{abstract}

\section{General Terms}

Integral absolute error (IAE), Integral of time absolute error (ITAE), FLC, FLC-GA

\section{Keywords}

Fuzzy logic controller, DC motor, Genetic algorithm, PI controller, Embedded fuzzy controller

\section{INTRODUCTION}

DC motor is a good choice for high performance motion control systems requiring four-quadrant operation including field weakening, minimum torque ripple, rapid speed recovery under impact load torque in addition to fast dynamic torque and speed responses. Development of a high performance controller to ensure competitive, robust and reliable speed or position controlled dc drive is a topic of interest of many researchers. The Proportionalcum-Integral (P-I) controller was so far the most widely used controller for dc drives [14].

Fuzzy logic, the logic of approximate reasoning, continues to grow in importance, as it provides an inexpensive solution for controlling ill-known complex systems. Fuzzy logic controllers [6] are already used in appliances, computer subsystems, industrial systems, automotive-related applications, consumer electronics, and so on. In last decade, fuzzy controllers have received adequate attention in motion control systems, as the later possess nonlinear characteristics, and a precise model is most often unknown. Fuzzy controller is already applied to phase controlled converter dc drive [9], linear servo drive [20], and induction motor drive [11-12].

The use of Fuzzy Logic [11] has found application in the area of control system design where human expert knowledge, rather than precise mathematical modelling, of a process or plant is used to model or implement the required controller. Human expert knowledge is based upon heuristic information gained in relation to the operation of the plant or process, and its inherent vagueness ("fuzziness") offers a powerful tool for the modelling of complex systems. Uncertainty and ambiguity are evident in many engineering problems. Fuzzy Logic Control (FLC) therefore provides a formal method of translating subjective and imprecise human knowledge into control strategies, thus facilitating better system performance through the exploitation and application of that knowledge. Optimal design of the FLC [8] Knowledge Base is central to the performance of the FLC. The rule base reflects the human expert knowledge, expressed as linguistic variables, while the membership functions represent expert interpretation of those variables. In the absence of such knowledge, a common approach is to optimize these FLC parameters through a process of trial and error, with respect to the performance of the system . This approach becomes impractical for systems having significant numbers of inputs since the rule-base size grows exponentially and consequently the number of rule combinations becomes significantly large.

The use of Genetic Algorithms (GA) [16] in this regard can provide such a solution. Genetic Algorithms (GA's) are robust, numerical search methods that mimic the process of natural selection. Although not guaranteed to absolutely find the true global optima in a defined search space, GA's are renowned for finding relatively suitable solutions within acceptable time frames and for applicability to many problem domains. Using primary concepts of genetic selection, crossover and mutation, GA's are a stochastic, but directed method of identifying global optima within a problem domain-space. This is achieved through successive performance testing of potential solutions, which collectively form a population, with respect to a problem objective function. In this way, convergence to optimal solutions is achieved.

The Knowledge Base of a Fuzzy Logic Controller (FLC) encapsulates expert knowledge and consists of the Data Base and Rule-Base of the controller. Optimization of both of these Knowledge Base components is critical to the performance of the controller and has traditionally been achieved through a process of trial and error. Such an approach is convenient for FLCs having low numbers of input variables, however for greater numbers of inputs, more formal methods of Knowledge Base optimization are required. Genetic Algorithms (GA's) provide such a method. They are 
stochastic, but directed, numerical search methods which use operators consistent with evolutionary theory and find application in many disciplines, particularly function optimization problems. Although not learning algorithms in the strictest sense, GA's can be applied to learning tasks. At last the GA based FLC has been applied to the control system of DC servo motor [19] by using digital signal processor (DSP) TMS320LF2407A. The controller improves the performance and the robustness of the servo system. In this paper, the mathematical model of DC motor has been extracted for the speed control. Three different models were designed using Proportional integral (PI), fuzzy logic and integrated Fuzzy logic -Genetic algorithm. The performance of the above models has been compared using transient, steady state parameters and error performance criterion.

\section{CASE STUDY}

In servo applications, a DC motor is required to produce rapid acceleration from stand still. Therefore the physical requirements of this kind of motor are low inertia, high starting torque and high starting current. Low inertia is achieved by reducing armature diameter with a consequent increase in armature length to achieve the desired power. The nomenclature used in the case study is given in Table I.

\subsection{Nomenclature}

Table 1. Nomenclature used in the case study

\begin{tabular}{|l|l|l|}
\hline $\mathrm{R}_{\mathrm{a}}$ & Resistance of armature & $2.1975 \Omega$ \\
\hline $\mathrm{L}_{\mathrm{a}}$ & Inductance of armature winding & $0.0063 \mathrm{H}$ \\
\hline $\mathrm{E}_{\mathrm{a}}$ & Applied Armature Voltage & $240 \mathrm{~V}$ \\
\hline $\mathrm{T}_{\mathrm{m}}$ & Torque developed by motor & $3 \mathrm{Nm}$ \\
\hline $\mathrm{J}$ & Moment of inertia & $0.0236 \mathrm{kgm}^{-2}$ \\
\hline $\mathrm{f}_{0}$ & Viscous friction coefficient & 0.015 \\
& & $(\mathrm{Nm} /(\mathrm{rad} / \mathrm{sec}))$ \\
\hline $\mathrm{K}_{\mathrm{m}}$ & Torque Constant & 1.78 \\
\hline $\mathrm{N}$ & Rated Speed & $1200 \mathrm{rpm}$ \\
\hline $\mathrm{P}$ & Rated Power & $120 \mathrm{~W}$ \\
\hline
\end{tabular}

\subsection{Mathematical Modelling}

Figure 1 represents the schematic diagram of armature controlled DC motor. Mathematical modelling of DC motor has been done to control the speed of the motor.

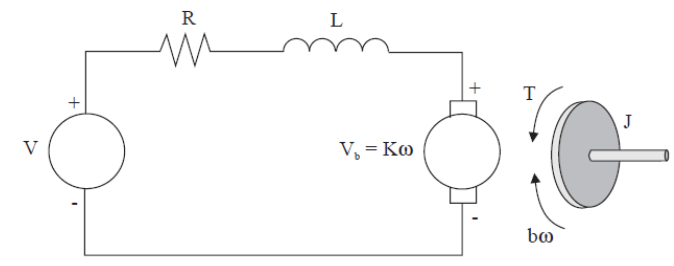

Figure 1. Armature Controlled DC Motor

The air gap flux is proportional to the field current.

$$
\begin{aligned}
& \phi \alpha I_{f} \\
& \Rightarrow \phi \phi=K_{f} I_{f}
\end{aligned}
$$

Torque is proportional to the product of armature current and air gap flux.

$T_{M}=K_{1} K_{f} I_{f} I_{a}$

$\Rightarrow T_{M}=K_{T} I_{a}\left(\mathrm{I}_{\mathrm{f}}\right.$ is constant for armature control $)$
Motor back EMF is proportional to speed.

$$
\begin{aligned}
& e_{b}=K_{b} \frac{d \theta}{d t} \\
& \Rightarrow e_{b}=K_{b} \omega(t)
\end{aligned}
$$

The differential equation for the armature circuit is

$L_{a} \frac{d I_{a}}{d t}+R_{a} I_{a}+e_{b}=e_{a}$

$\Rightarrow L_{a} \frac{d I_{a}}{d t}+R_{a} I_{a}+K_{b} \omega(t)=e_{a}$

$\Rightarrow \frac{d I_{a}}{d t}=-\frac{R_{a}}{L_{a}} I_{a}(t)-\frac{K_{b}}{L_{a}} \omega(t)+\frac{E_{a}}{L_{a}}$

The torque equation is

$J \frac{d^{2} \theta}{d t^{2}}+f_{0} \frac{d \theta}{d t}=T_{M}(t)$

$\Rightarrow J \frac{d^{2} \theta}{d t^{2}}+f_{0} \frac{d \theta}{d t}=K_{T} I_{a}$

$\Rightarrow J \frac{d \omega(t)}{d t}+f_{0} \frac{d \omega(t)}{d t}=K_{T} I_{a}$

$\Rightarrow \frac{d \omega(t)}{d t}=\frac{K_{T}}{J} I_{a}(t)-\frac{f_{0}}{J} \omega(t)$

The state space equations are

$\dot{x}(t)=A x(t)+B u(t)$

$y(t)=C x(t)$

The state space representation of DC motor model is $\left[\begin{array}{c}\dot{x}_{1}(t) \\ \dot{x}_{2}(t)\end{array}\right]=\left[\begin{array}{cc}-\frac{f_{0}}{J} & \frac{K_{T}}{J} \\ -\frac{K_{b}}{L_{a}} & -\frac{R_{a}}{L_{a}}\end{array}\right]\left[\begin{array}{c}x_{1}(t) \\ x_{2}(t)\end{array}\right]+\left[\begin{array}{c}0 \\ \frac{1}{L_{a}}\end{array}\right] u(t)$

$y(t)=x_{1}(t)$

$\left(J s^{2}+f_{0} s\right) \theta(s)=T_{M}(s)$

$\left(J s^{2}+f_{0} s\right) \theta(s)=K_{T} I_{a}(s)$

$\frac{\theta}{E_{a}}(s)=\frac{K_{T}}{s\left[\left(R_{a}+s L_{a}\right)\left(J s+f_{0}\right)+K_{T} K_{B}\right]}$

$\frac{\theta}{E_{a}}(s)=\frac{\frac{K_{T}}{R_{a}}}{J s^{2}+s\left(f_{0}+\frac{K_{T} K_{b}}{R_{a}}\right)}$ 


$$
\begin{aligned}
& \frac{\theta}{E_{a}}(s)=\frac{\frac{K_{T}}{R_{a}}}{s(J s+f)} \\
& \frac{\theta}{E_{a}}(s)=\frac{K_{m}}{s\left(s \tau_{m}+1\right)} \\
& K_{m}=\frac{K_{T}}{R_{a} f} \\
& \tau_{m}=\frac{J}{f} \\
& G_{a}(s)=\frac{\theta(\mathrm{s})}{V(s)}=\frac{K}{s\left[(R+L s)(J s+b)+K^{2}\right]} \\
& G_{v}(s)=\frac{\omega(\mathrm{s})}{V(s)}=\frac{K}{\left[(R+L s)(J s+b)+K^{2}\right]}
\end{aligned}
$$

This case study of DC servo motor is tested by a simulation study considering real conditions to be able to occur in real-time running of the system using MATLAB Simulink. Figure 2 shows the representation of DC model in Simulink.

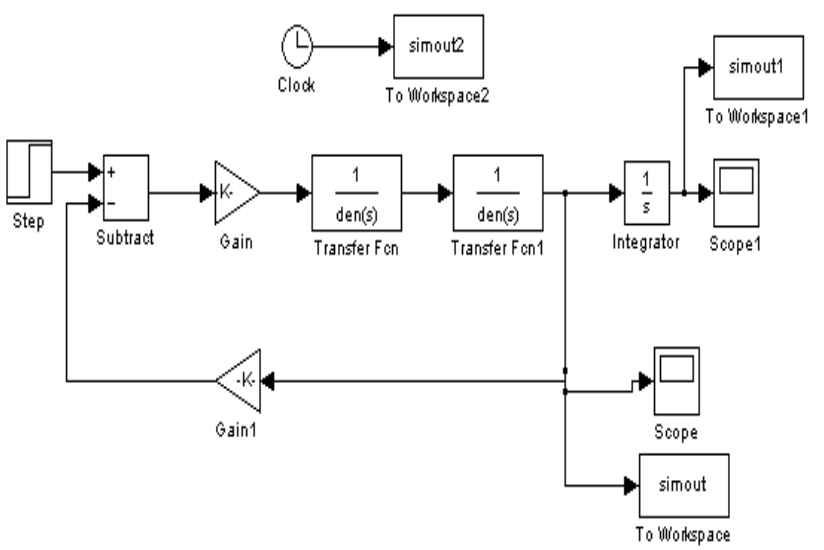

Figure 2. Simulink representation of DC motor model

Figure 3 shows the Angular speed of DC motor and figure 4 shows the angle of DC motor.

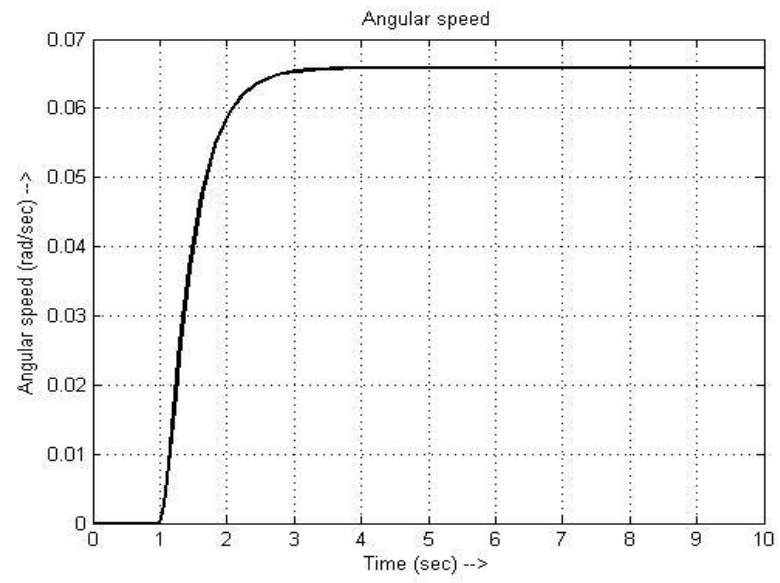

Figure 3. Graph for angular speed w ( rad/sec) of DC motor

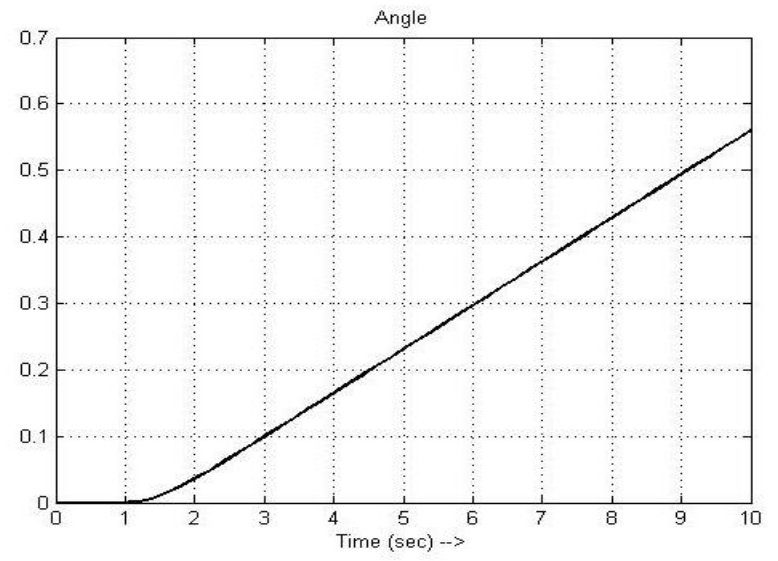

Figure 4. Graph for angle of DC motor

\section{PI CONTROLLER DESIGN}

There are different speed control techniques for DC motor.

1. Field control method

2. Armature resistance control method

3. Armature voltage control method

This case study looks in to armature voltage speed control where armature voltage of the DC motor is varied to control the speed of the motor. Table II shows the parameters of the tested motor and Table III shows the experimental data obtained from the laboratory for the speed and rotor current variation for the different armature voltages.

Table 2. Parameters of tested motor

\begin{tabular}{|c|c|}
\hline Power & $120 \mathrm{~W}$ \\
\hline Rotate Speed & $1200 \mathrm{rpm}$ \\
\hline
\end{tabular}

Table 3. Experimental data

\begin{tabular}{|c|c|c|}
\hline Armature Voltage & Rotor Current & Speed \\
\hline 210 & 0.515 & 0.139 \\
\hline 213 & 0.51 & 0.191 \\
\hline 215 & 0.53 & 0.244 \\
\hline 217 & 0.51 & 0.296 \\
\hline 218 & 0.48 & 0.34 \\
\hline 224 & 0.4 & 0.366 \\
\hline 225 & 0.38 & 0.4 \\
\hline
\end{tabular}

The schematic diagram of the dc motor system is shown in figure 6 . The power circuit consists of a three phase, fully controlled bridge converter that drives a separately excited dc motor. For simplicity, the converter drive system is used in motoring mode only with fixed field excitation. Inner current control loop is provided in addition to the speed control loop to achieve fast transient response as well as to limit the armature current. The output of the current controller is the control voltage $\mathrm{V}_{\mathrm{c}}$ for the converter firing circuit. The firing pulses for the SCRs are generated with a delay angle, by cosine wave crossing method. The speed and current controllers in figure 6 may be P-I controllers or fuzzy logic controllers. The design methodology for both type of controllers are discussed below. Figure 5 shows the proposed scheme for speed control of armature controlled DC motor. 


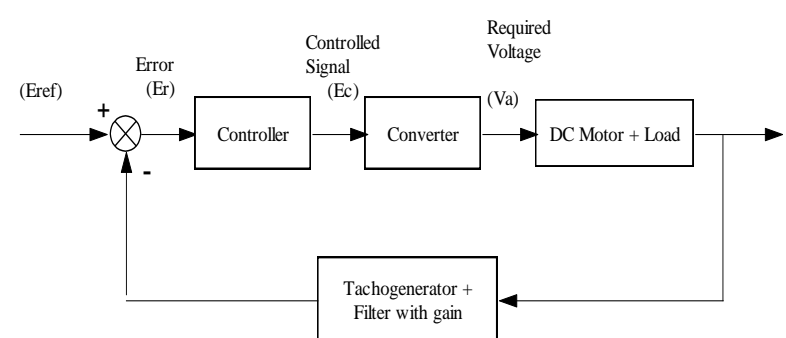

Figure 5. Proposed scheme for speed control of armature controlled DC motor

Figure 6 shows the Detailed control scheme of speed control of DC motor

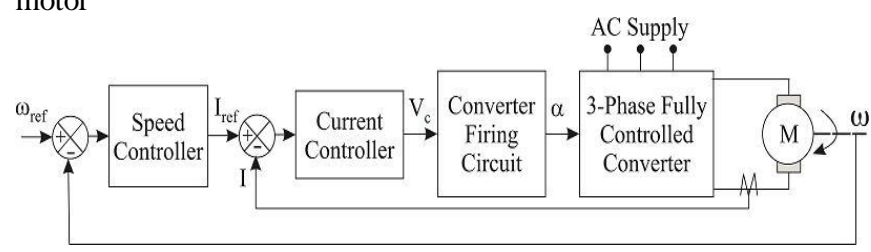

Figure 6. Detailed control scheme of speed control of DC motor

Figure 7 shows the Circuit diagram of 3 phase fully controlled converter used in motor.

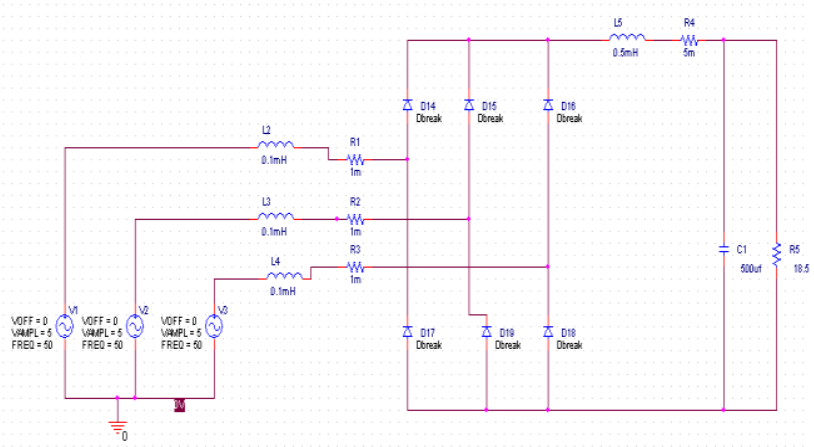

Figure 7. Circuit diagram of $\mathbf{3}$ phase fully controlled converter

Figure 8 shows the 3 phase input power supply to the motor.

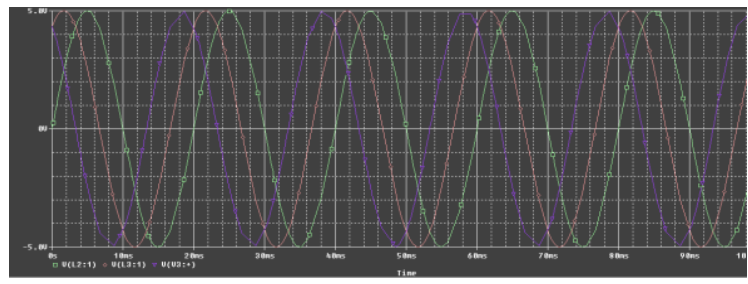

Figure 8. 3 Phase input power supply

Figure 9 shows the Converter output.

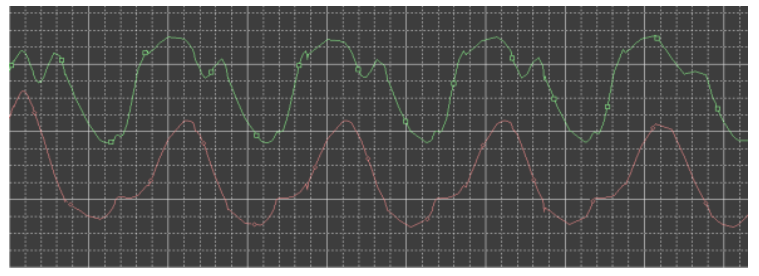

Figure 9. Converter output.
Figure 10 shows the Block diagram schematic of speed control of DC motor

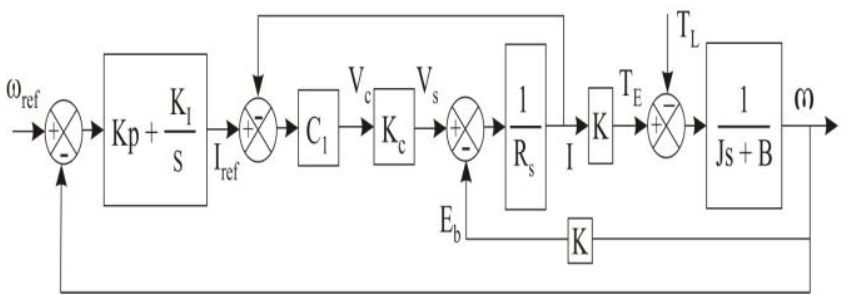

Figure 10. Block diagram schematic of speed control of DC motor

\section{DESIGN OF FUZZY LOGIC CONTROLLER}

PID controller [1] is a standard control structure for classical control theory. But the performance is greatly distorted and the efficiency is reduced due to nonlinearity in the process plant. The fuzzy PID controllers are the natural extension of their conventional version, which preserve their linear structure of PID controller. The fuzzy PID controllers are designed using fuzzy logic control principle in order to obtain a new controller that possesses analytical formulas very similar to digital PID controllers. Fuzzy PID controllers have variable control gains in their linear structure. These variable gains are nonlinear function of the errors [2] and changing rates of error signals. The main contribution of these variable gains in improving the control performance is that they are self- tuned and they can adapt to rapid changes of the errors and rate of change of error caused by time delay effects, nonlinearities and uncertainties of the underlying process.

Figure 11 shows the basic architecture of fuzzy logic controller

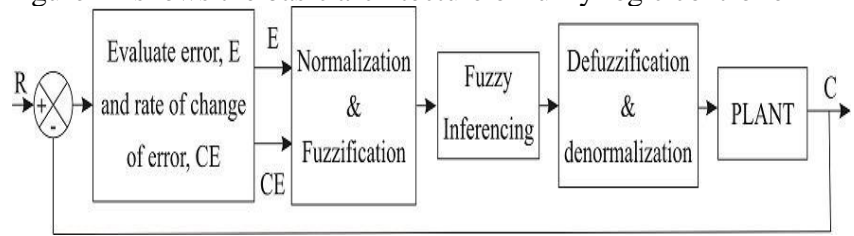

Figure 11. Basic architecture of fuzzy logic controller

In this paper different linguistic variables have been considered. Table IV shows the different linguistic variables for fuzzy logic controller.

Table 4. Linguistic variable of fuzzy logic control

\begin{tabular}{|l|l|l|l|l|l|}
\hline \multicolumn{2}{|c|}{ Error e(t) } & \multicolumn{2}{c|}{$\begin{array}{c}\text { Change in error } \\
\text { At) }\end{array}$} & \multicolumn{2}{c|}{$\begin{array}{c}\text { Controller output } \\
\text { u(t) }\end{array}$} \\
\hline NB & $\begin{array}{l}\text { Negative } \\
\text { Big }\end{array}$ & NB & Negative Big & NB & $\begin{array}{l}\text { Negative } \\
\text { Big }\end{array}$ \\
\hline NM & $\begin{array}{l}\text { Negative } \\
\text { Medium }\end{array}$ & NM & $\begin{array}{l}\text { Negative } \\
\text { Medium }\end{array}$ & NM & $\begin{array}{l}\text { Negative } \\
\text { Medium }\end{array}$ \\
\hline NS & $\begin{array}{l}\text { Negative } \\
\text { Small }\end{array}$ & NS & $\begin{array}{l}\text { Negative } \\
\text { Small }\end{array}$ & NS & $\begin{array}{l}\text { Negative } \\
\text { Small }\end{array}$ \\
\hline ZO & Zero & ZO & Zero & ZO & Zero \\
\hline PS & $\begin{array}{l}\text { Positive } \\
\text { Small }\end{array}$ & PS & $\begin{array}{l}\text { Positive } \\
\text { Small }\end{array}$ & PS & $\begin{array}{l}\text { Positive } \\
\text { Small }\end{array}$ \\
\hline PM & $\begin{array}{l}\text { Positive } \\
\text { Medium }\end{array}$ & PM & $\begin{array}{l}\text { Positive } \\
\text { Medium }\end{array}$ & PM & $\begin{array}{l}\text { Positive } \\
\text { Medium }\end{array}$ \\
\hline PB & Positive Big & PB & Positive Big & PB & Positive Big \\
\hline
\end{tabular}

Designing a good fuzzy rule base is the key to obtain satisfactory control performance for a particular operation. Classical analysis 
and control strategy are incorporated in the rule base. The rule base used in simulation is summarized in Table IV. Each rule has the form IF e $(t)$ is NB AND $\Delta e(t)$ is NB THEN $u(t)$ is NB. The control literature has worked towards reducing the size of the rule base and optimizing the rule base using different optimization techniques like Genetic Algorithms, Pattern Swarm Optimization [3] for intelligent controller.

The universe of discourse of input variables and the output are divided from -6 to +6 . Each universe of discourse is divided into seven fuzzy sets: NB, NM, NS, Z, PS, PM and PB. Each fuzzy variable is a member of the subsets with a degree of between 0 (non member) and 1(full member).Figure 12 shows the Membership functions of inputs and output
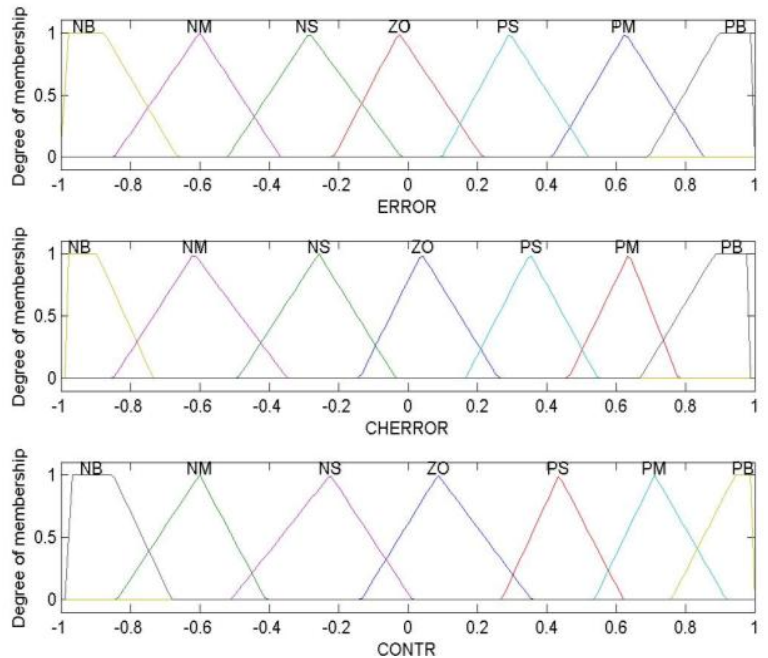

Figure 12. Membership functions of inputs and output

The fuzzy rules are actually experience rules based on expertise or operator's long-time experiences. Table 5 shows the fuzzy rules for the case study. The variables are processed by an inference engine which executes 49 rules $(7 * 7)$.

Table 5 shows Rule base for Fuzzy logic controller

Table 5. If-then rule base for fuzzy logic control

\begin{tabular}{|c|c|c|c|c|c|c|c|c|}
\hline \multirow{2}{*}{\multicolumn{2}{|c|}{$\mathbf{u}(\mathbf{t})$}} & \multicolumn{7}{|c|}{$\mathbf{e}(\mathbf{t})$} \\
\hline & & NB & NM & NS & $\mathrm{ZO}$ & PS & $\mathbf{P M}$ & $\overline{\text { PB }}$ \\
\hline \multirow{7}{*}{$\Delta \mathbf{e}(\mathbf{t})$} & NB & NB & NB & NB & NB & $\mathrm{NM}$ & NS & $\mathrm{ZO}$ \\
\hline & NM & NB & NB & NB & NM & NS & $\mathrm{ZO}$ & PS \\
\hline & NS & NB & NB & $\mathrm{NM}$ & NS & $\mathrm{ZO}$ & PS & PM \\
\hline & $\mathbf{Z O}$ & NB & NM & NS & $\mathrm{ZO}$ & PS & PM & PB \\
\hline & PS & NM & NS & $\mathrm{ZO}$ & PS & PM & PB & PB \\
\hline & PM & NS & $\mathrm{ZO}$ & PS & PM & PB & PB & PB \\
\hline & PB & $\mathrm{ZO}$ & PS & $\mathrm{PM}$ & PB & PB & PB & PB \\
\hline
\end{tabular}

At last, defuzzified output is obtained from the fuzzy inputs. In this research work centroid method of de-fuzzification is used. It is expressed in Equation.13.

$$
u^{*}=\frac{\int \mu_{c}(u) * u d u}{\int \mu_{c}(u) d u}
$$

\section{OPTIMIZATION USING GENETIC ALGORITHM (FLC-GA APPROACH)}

Genetic Algorithms are intelligent optimization technique that relies on the parallelism found in nature; in particular its searching procedures are based on the mechanics of natural selection and genetics. GAs was first conceived in the early 1970s by Holland [19]. GAs is used regularly to solve difficult search, optimization, and machine-learning problems that have previously resisted automated solutions. They can be used to solve difficult problems quickly and reliably. These algorithms are easy to interface with existing simulations and models, and they are easy to hybridize. GAs includes three major operators: selection, crossover, and mutation, in addition to four control parameters: population size, selection crossover and mutation rate. This paper is concerned primarily with the selection and mutation operators. There are three main stages of a genetic algorithm; these are known as reproduction, crossover and mutation [16]. The flow chart of a genetic algorithm is shown in figure 6. The flow chart of genetic algorithm is described below.

[Start] Generate random population of $n$ chromosomes (suitable solutions for the problem).

[Fitness] Evaluate the fitness $f(x)$ of each chromosome $x$ in the population.

[New population] Create a new population by repeating following steps until the new population is complete.

a) Selection. Select two parent chromosomes from a population according to their fitness (the better fitness, the bigger chance to be selected).

b) Crossover. With a crossover probability, cross over the parents to form new offspring (children). If no crossover was performed, offspring is the exact copy of parents.

c) Mutation. With a mutation probability, mutate new offspring at each locus (position in chromosome).

d) Accepting. Place new offspring in the new population.

[Replace] Use new generated population for a further run of the algorithm.

[Test] If the end condition is satisfied, stop, and return the best solution in current population.

[Loop] Go to step 2.

The objective functions are MSE (Mean Square Error), IAE (Integral Absolute Error), ISE (Integral Square Error) and ITAE (Integral Time Absolute Error). The main objective of PID controller is to minimize the error signal or in other words we can say that minimization of performance indices.

$$
\begin{aligned}
& \text { MSE }=\frac{1}{t} \int_{0}^{t}(e(t))^{2} \\
& \text { IAE }=\int_{0}^{t}|e(t)| d t \\
& \text { ISE }=\int_{0}^{t}|e(t)|^{2} d t \\
& \text { ITAE }=\int_{0}^{t} t|e(t)| d t
\end{aligned}
$$


Figure 13 shows the Flow chart of genetic algorithm

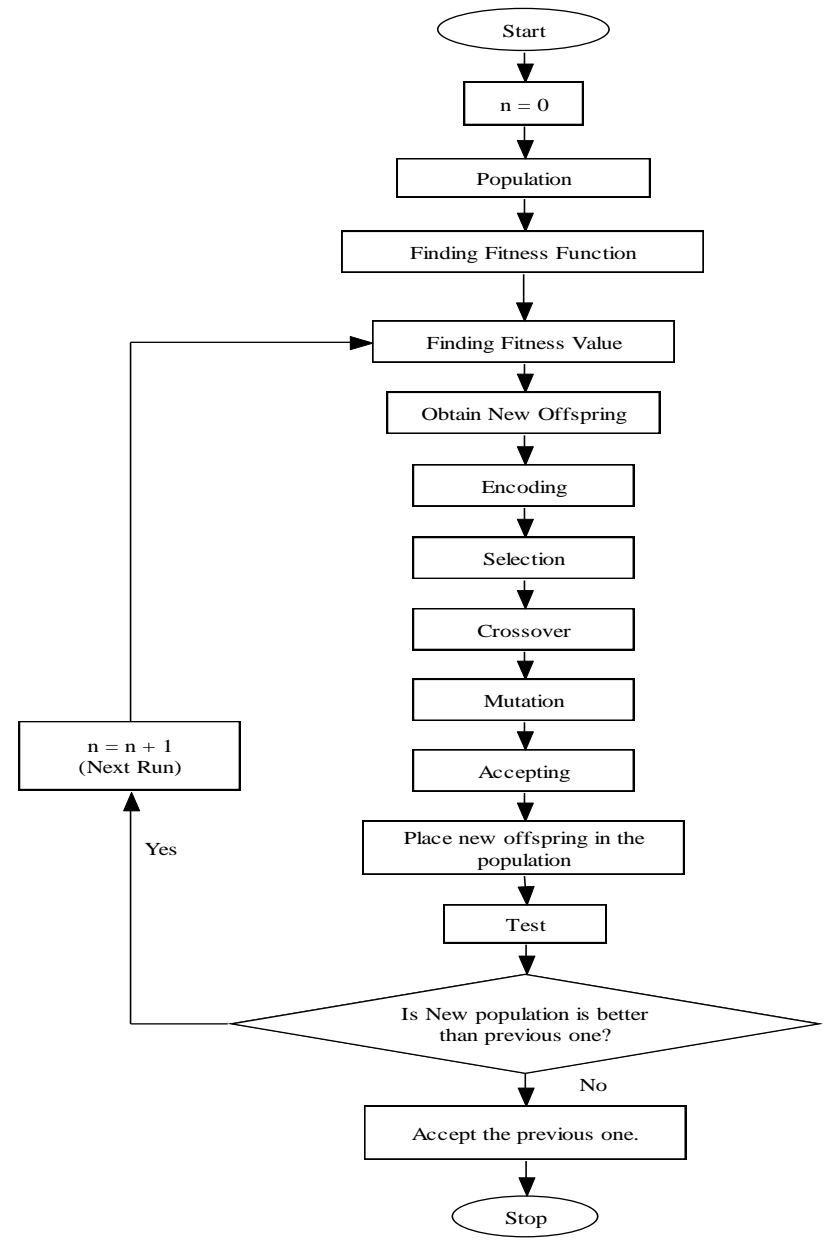

Figure 13. Flow chart of Genetic Algorithm

The fitness value of the chromosome is the inverse of the performance indices. The fitness value is used to select the best solution in the population to the parent and to the offspring that will comprise the next generations. The fitter the parent greater is the probability of selection. This emulates the evolutionary process of "survival of the fittest". Parents are selected using roulette wheel selection method.

Fitness function is reciprocal of performance indices. In this paper we have taken the discrete form of ITAE. ITAE is treated as performance indices and fitness function denoted by $\mathrm{J}$ can be described below in Equation 18.

$$
J=\frac{1}{\sum_{k=0}^{N-1}[\{r(k T)-c(k T)\} T]}
$$

\section{EMBEDDED FUZZY CONTROLLER}

This section describes the embedded fuzzy logic controller using high end microcontroller unit and using Digital Signal Processor system. This paper discusses the real time embedded control using ST6MCU (ST6265) and using digital signal processor TMS320LF2407A. [17]

Practical schematic of the universal motor speed control with fuzzy logic using Digital Signal Processor is shown in figure 14. The fuzzy logic regulation loop is achieved by a standard ST6 MCU. The motor is supplied in DC mode through a chopper stage composed of an IGBT STGP10N50 and a freewheeling diode STTA806DI. The board is directly connected on the mains.

The speed control scheme is shown in figure 13 . The TMS320LF2407A DSP calculates $[4,10]$ the present speed by counting the pulses form the incremental encoder attached to motor shaft. The calculated speed is then compared with set point speed. The error and change of error are the two inputs to which are Fuzzified for Fuzzy controller. The set of rules are defined in fuzzy inference engine which gives Fuzzified output depending on the number of rules fired. According to the defuzzified output it varies the PWM duty cycles by changing its Compare register 1 (CMPR1) contents. It has Event Manager that is specially designed for the motor control and motion control applications. The general-purpose Timer 1 in EVA (Event Manager A) is used in continuous up/down count mode for the symmetric PWM generation. The generalpurpose Timers 3 and 4 of EVB (Event Manager B) are used for the speed measurement purpose. The PWM output coming from the DSP is $3.3 \mathrm{~V}$. This voltage is fed to the MOSFET driver for giving PWM input to the gate of MOSFET. The controlled output voltage from the buck converter is given as the input to the DC motor. According to the given input voltage the motor starts rotating. The incremental encoder connected to the motor gives pulses which are fed to counter in DSP processor. The Event Manager B (EVB) is used for the speed measurement purpose. The timer 3 is configured for some periodic interrupt. The timer 4 is configured to have the optical encoder pluses as the external clock source and its counter is set to continuous UP count mode. Timer 4 is synchronized with timer 3. The value of the timer 4 counter is taken and is converted to speed. This gives the present speed in rpm. According to the difference between the required speed and the present speed the CMPR1 register content is modified by the Fuzzy controller algorithm. The GA based fuzzy controller is realized by using TMS320LF2407A DSP, which gives a good performance. Figure 15,16 shows the voltage curve of DC servo motor by using the PID controller and GA based fuzzy controller, which indicates that the voltage wave shape of the servo motor is steadier by using integrated Fuzzy -GA controller than PID controller.

Figure 14 shows the Speed control scheme using digital signal processor TMS320LF2407A

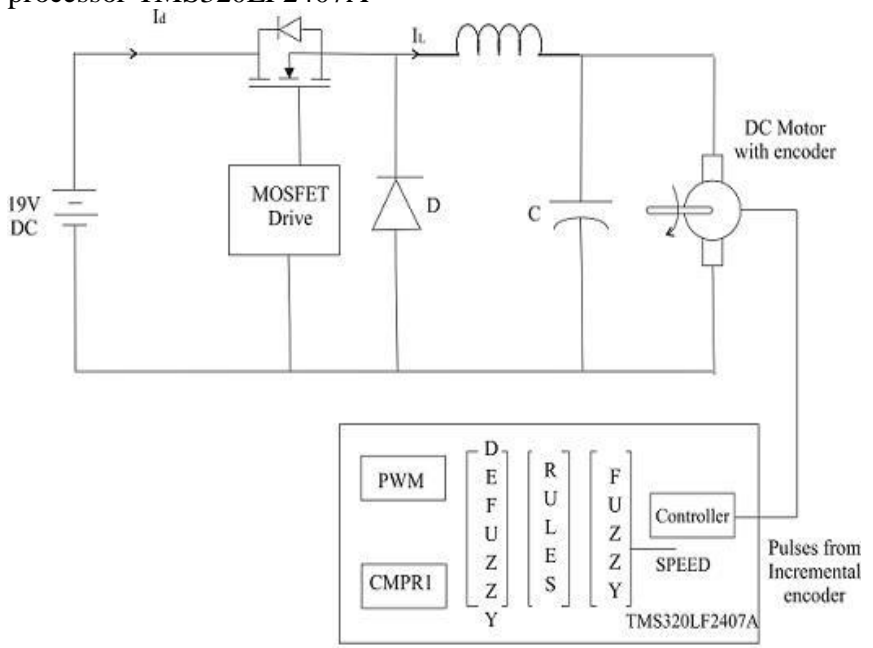

Figure 14. Speed control scheme using digital signal processor TMS320LF2407A 


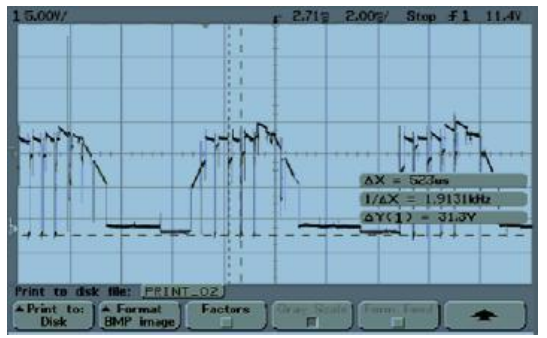

Figure 15. Voltage curve using conventional PI controller

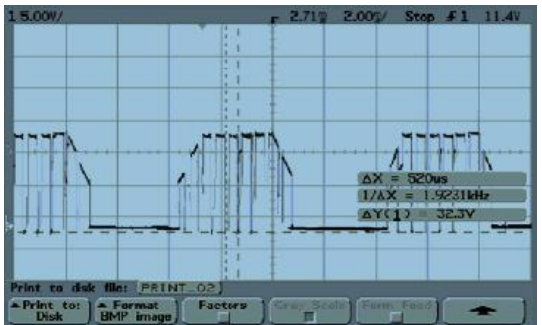

Figure 16. Voltage curve using DSP TMS320LF2407A controller

\section{SIMULATION STUDIES}

The designed optimal control system has been simulated via MATLAB Simulink software. In order to illuminate the performance of fuzzy GA controller, conventional fuzzy controller and PI controller is used as comparison with it. Simulink representations of DC motor with PI controller, fuzzy logic controller and fuzzy GA controller have been implemented and investigated.

Figure 17 shows the Simulink representation of DC motor with PI controller.

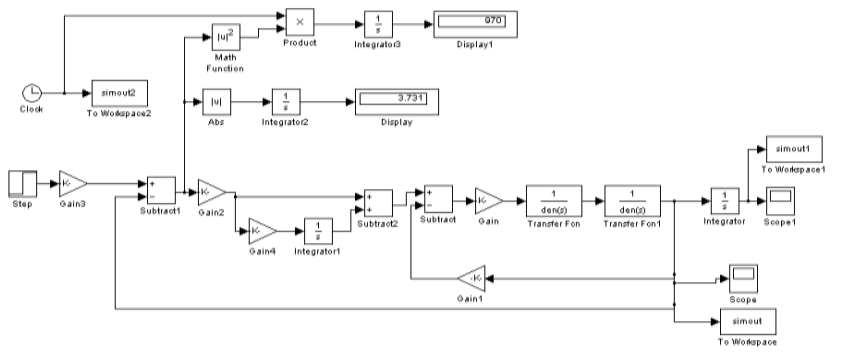

Figure 17. Simulink representation of DC motor with PI controller

Figure 18 represents the step response for speed control of DC motor using PI controller.

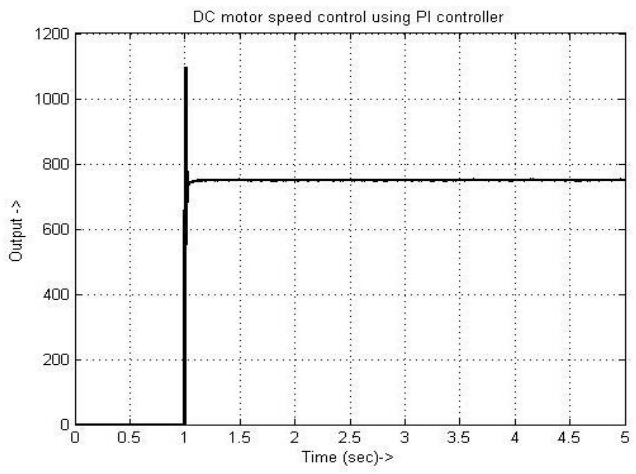

Figure 18. Step response of speed control of DC motor using PI controller
Figure 19 shows the representation of the DC motor using Fuzzy logic controller.

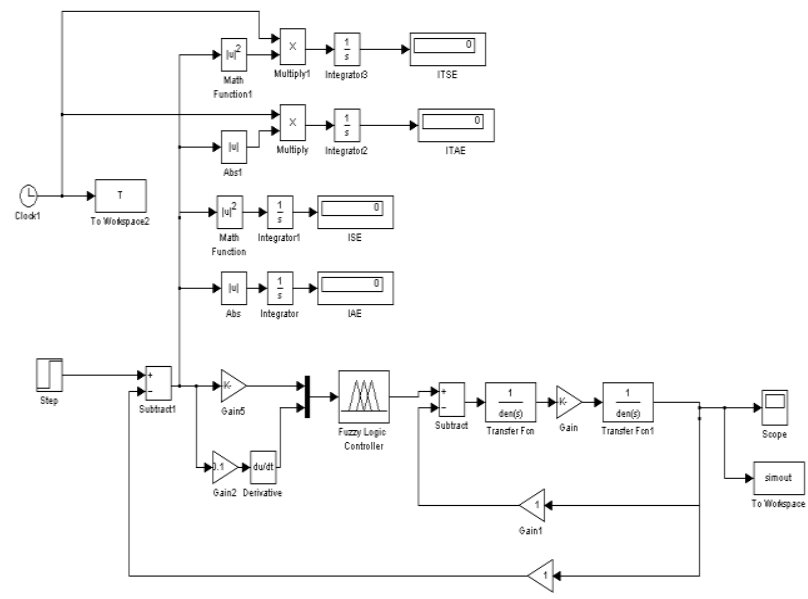

Figure 19. Simulink representation of DC motor using fuzzy logic controller

Figure 20 Shows the Step response of speed control of DC motor using Fuzzy Logic Controller. The fuzzy logic controller gives much lower overshoot than conventional PI controller.

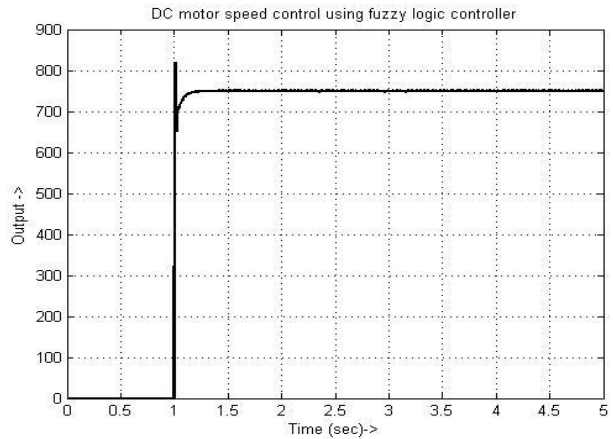

Figure 20. Step response of speed control of DC motor using Fuzzy Logic Controller

Figure 21 Shows the Simulink representation of DC motor using fuzzy-GA controller.

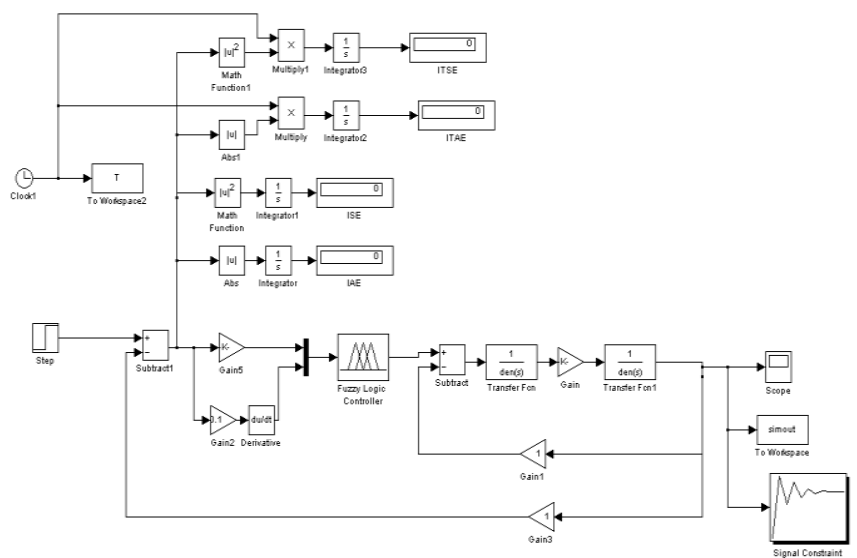

Figure 21. Simulink representation of DC motor using fuzzyGenetic Algorithm

Figure 22 shows the step response of fuzzy-GA system which is better optimized than fuzzy logic controller and PI controller. 


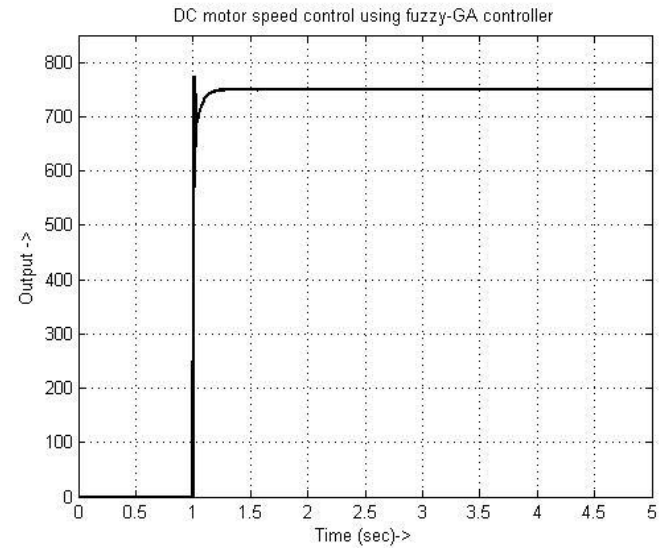

Figure 22. Step response of speed control of DC motor using fuzzy-Genetic Algorithm

Figure 23 shows the Surface plot of FIS.

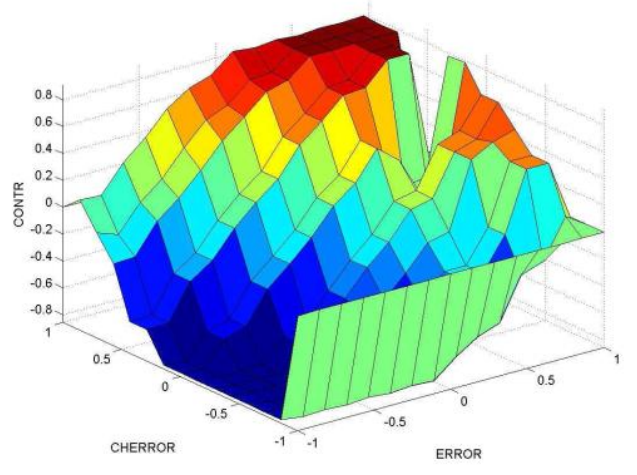

Figure 23. Surface plot of FIS.

\section{RESULTS AND DISCUSSIONS}

In order to verify the validity of the integrated fuzzy GA controller, various parameters like Maximum overshoot, settling time, peak time of conventional PI controller and fuzzy logic controller have been compared with GA based FLC. The parameters taken up for study are mathematically expressed as under.

$$
\begin{aligned}
& \% M_{p}=e^{\frac{-\pi \xi}{\sqrt{1-\xi^{2}}}}, \\
& t_{p}=\frac{\pi}{\omega_{n} \sqrt{1-\xi^{2}}}, \\
& t_{r}=\frac{\pi-\tan ^{-1}\left(\frac{\sqrt{1-\xi^{2}}}{\xi}\right)}{\omega_{n} \sqrt{1-\xi^{2}}}
\end{aligned}
$$

Table VI shows the comparison of various parameters which shows the PI controller has bigger overshoot. In comparison with PI controller the Fuzzy logic controller shows much low overshoot which is further optimized by integrated FLC-GA controller which shows only about $3.2 \%$ of maximum overshoot. Similarly the settling time and peak time reduction in FLC_GA controllers shows that the integrated fuzzy-GA approach is more effective and efficient than conventional methods. Figure 24, 25 and 26 shows the performance comparison of the various parameters for different types of controllers.

Table 6. Comparison of steady state and transient state parameters of PID controller, Fuzzy controller and Fuzzy-GA controller

\begin{tabular}{|l|l|c|c|c|}
\hline S.No & Controllers & \multicolumn{3}{|c|}{ Parameters } \\
\cline { 3 - 5 } & $\begin{array}{l}\text { Max. } \\
\text { Overshoot }\end{array}$ & $\begin{array}{l}\text { Settling } \\
\text { Time }\end{array}$ & $\begin{array}{l}\text { Peak } \\
\text { Time }\end{array}$ \\
\hline $\mathbf{1}$ & PI Controller & $45.33 \%$ & $2.5 \mathrm{Sec}$. & 3.1 Sec. \\
\hline $\mathbf{2}$ & $\begin{array}{l}\text { Fuzzy Logic } \\
\text { Controller }\end{array}$ & $9.33 \%$ & $1.8 \mathrm{Sec}$. & $2.3 \mathrm{Sec}$. \\
\hline $\mathbf{3}$ & Fuzzy-GA & $3.2 \%$ & $1.5 \mathrm{Sec}$. & $1.7 \mathrm{Sec}$. \\
\hline
\end{tabular}

MAXIMUM OVERSHOOT

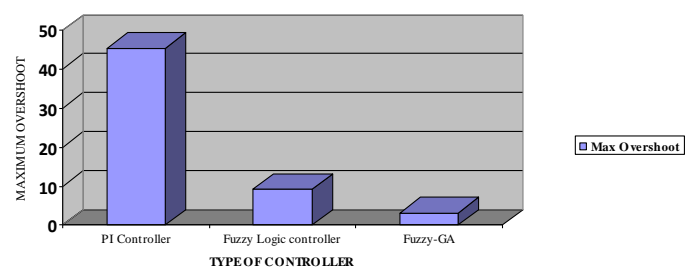

Figure 24. Comparison of Maximum Overshoot for conventional, FLC and FLC-GA controller

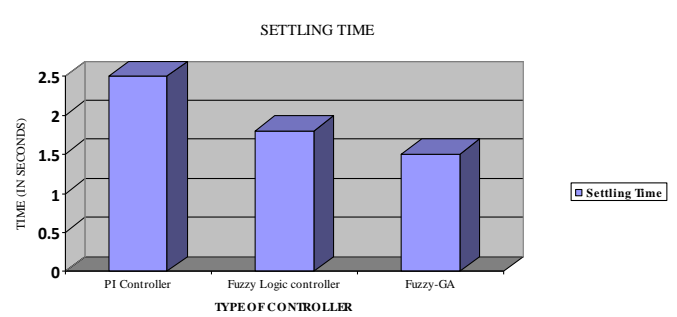

Figure 25. Comparison of Settling time for conventional, FLC and FLC-GA controller

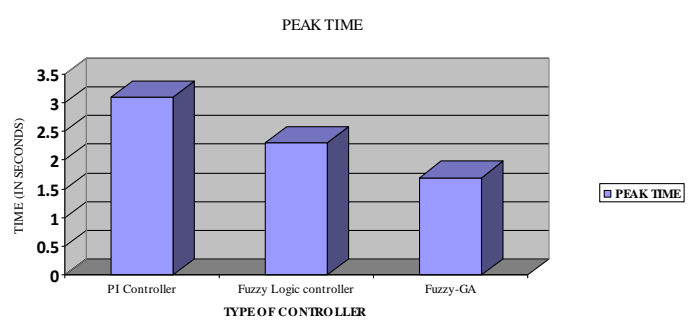

Figure 26. Comparison of Peak Time for conventional, FLC and FLC-GA controller

Table VII gives the comparative study of the performance parameters IAE and ITAE for the different types of controllers. It has been noted that the error in integrated fuzzy-GA controller is minimum when compared to other controllers which shows the superiority of hybridization.

Table 7. Comparison of performance indices parameters of PID controller, fuzzy controller and fuzzy-GA controller

\begin{tabular}{|l|l|c|c|}
\hline S.No & \multirow{2}{*}{ Parameters } & \multicolumn{2}{|c|}{ Performance criteria } \\
\cline { 3 - 4 } & & IAE & ITAE \\
\hline 1 & PI Controller & 0.03195 & 0.004 \\
\hline 2 & Fuzzy Logic Controller & 0.012 & 0.003 \\
\hline 3 & Fuzzy-GA & 0.08 & 0.001 \\
\hline
\end{tabular}


This shows the superiority of fuzzy-GA hybridization over conventional techniques. The lowest overshoot, settling time and peak time of fuzzy GA controller have been noted in comparison with FLC and conventional PI controller. The performance improvement shows the superiority of hybridisation over conventional techniques.

\section{CONCLUSION}

This paper discusses a process control case study taking armature controlled DC motor. The speed of the DC motor is controlled using different controllers and real time embedded speed control techniques using fuzzy based MCU and digital signal processor is discussed. First of all a mathematical model of the system is developed and a conventional PI controller is implemented in it. The PI controller gives a very high overshoot and high settling time. So we proposed and implemented artificial intelligence principles in the controller architecture. Then we implemented a fuzzy logic control and optimized its step response using genetic algorithm. The fuzzy logic control gives a much better response than the conventional PI controller and optimization using genetic algorithm in the process gives an optimized response and an optimal control.

\section{REFERENCES}

[1] Atef Saleh Othman Al-Mashakbeh, "PID Control of DC Motor," European Journal of Scientific Research, Vol. 35, No. 2, 2009, pp. 198-203.

[2] B Allaoua, B Gasbaoui, B Mebarki, "Setting up PID DC motor speed control alteration parameters using PSO strategy," Leonardo Electronic Journal of Practices and technologies, 2009, pp. 19-32.

[3] Boumediène ALLAOUA, Brahim GASBAOUI and Brahim MEBARKI, "Setting Up PID DC Motor Speed Control Alteration Parameters Using Particle Swarm Optimization Strategy", Leonardo Electronic Journal of Practices and Technologies, Issue 14, January-June 2009, pp. 19-32

[4] Bhim Singh, B P Singh and (Ms) K Jain, "Implementation of DSP Based Digital Speed Controller for Permanent Magnet Brushless de Motor", IE(I) Journal-EL, Vol 84, June 2003, pp.16-21.

[5] B Mrozek, Z Mrozek, "Modeling and Fuzzy Control of DC Drive," in Proceedings of 14th European Simulation Multi conference ESM 2000, pp 186-190.

[6] C. C. Lee, "Fuzzy logic in control systems: fuzzy logic controller, part- I", IEEE Trans. on Systems, Man and Cybernet, vol. 20, no. 2, pp. 404- 418, Mar./Apr. 1990.

[7] C.-K. Lee, and W.-H. Pang, "A Brushless DC Motor Speed Control System Using Fuzzy Rules," Power Electronics and Variable-Speed Drives Conference, pp. 101-106, Oct 1994.

[8] Elke Laubwald, "Servo Control Systems: DC servo mechanisms", citation from control-systems-principles.co.uk.

[9] G.C.D. Sousa, and B. K. Bose, "A fuzzy set theory based control of a phase-controlled converter dc machine drive," IEEE Trans. on Industry Applications, vol. 30, no. 1, pp. 3444, Jan/Feb 1994.

[10] G.Madhusudhana Rao, B.V.SankerRam, B.Smapath Kumar and K.Vijay Kumar, "Speed Control of BLDC Motor Using DSP”, International Journal of Engineering Science and Technology, Vol. 2(3), 2010, pp.143-147.
[11] K. B. Mohanty, A. Routray, and N. K. De, "Design of a fuzzy sliding mode controller for a field oriented induction motor drive," Journal of Systems Society of India, 'Paritantra', vol. 6, no. 1, pp. 8-16, Aug. 2001.

[12] K. B. Mohanty, "Design and comparative study of sliding mode and fuzzy sliding mode controllers for an induction motor drive", Procc. of 25th National Systems Conf., Dec. 2001, Coimbatore, pp 66-71.

[13] Mehmet Akar and Ismail Temiz, "Motion controller design for the speed control of dc servo motor", International Journal of Applied Mathematics and Informatics, Issue 4, Volume 1, 2007, pp.131-137.

[14] P. K. Nandam, and P. C. Sen, "A comparative study of proportionalintegral (P-I) and integralproportional (I-P) controllers for dc motor drives," Int. Jour. of Control, vol. 44, pp. 283-297, 1986.

[15] P.-T. Chan, A.-B. Rad, and K.-M. Tsang, "Optimization of fused fuzzy systems via genetic algorithms," Trans. Ind. Electron of IEEE, Vol. 49, no. 3, pp. 685-692, 2002.

[16] Rahul Malhotra, Narinder Singh, Yaduvir singh, "Non-linear and optimal direct control of AC Drive using fuzzy-GA architecture", International journal of computer science and network security, August, 2009, Vol.9, No.8, pp.209-215.

[17] W.-S. Oh, Y.-T. Kim, C.-S. Kim, T.-S. Kown, and H.-J. Kim, "Speed Control of Induction Motor Using Genetic Algorithm Based Fuzzy Controller," Industrial Electronics Society Conference of IEEE, vol. 3, no. 2, pp. 625-629, 1999.

[18] Wai Phyo Aung, "Analysis on Modeling and Simulink of DC Motor and its Driving System Used for Wheeled Mobile robot," World Academy of Science, engineering and technology, 32, 2007, pp. 299-306.

[19] XiaChangliang, Guo Peijian, Shi Tingna and Wang Mingchao, "Speed control of brushless DC motor using genetic algorithm based fuzzy controller", Proceedings of the International Conference on Intelligent Mechatronics and Automation Chengdu,China August 2004, pp.460-464.

[20] Y.H. Bharathi, B.R. Rekha, P. Bhaskar, C.S. Parvathi and A.B. Kulkarni, "Multi-input Fuzzy Logic Controller for Brushless dc Motor Drives", Defence Science Journal, Vol. 58, No. 1, January 2008, pp. 147-158.

[21] Y. F. Li, and C. C. Lau, "Development of fuzzy algorithms for servo systems," IEEE Control System Magazine, vol. 9, no. 3, pp. 65-72, 1989.

[22] Yesil Engin and Erenoglu Isin, "An intelligent hybrid fuzzy PID controller", Proceedings of the $20^{\text {th }}$ European conference on Modelling and Simulation, Wolfgang Borutzky.

[23] Yager R. R. and Filer D. P., "Essentials of Fuzzy Modeling and Control, john Wiley, 1994.

[24] Yesil E., Guzelkaya M., Eksin I., "Fuzzy PID Controllers: An Overview", The third triennial ETAI International Conference on Applied Automatic Systems, skopje, Marcedonia, pp.105112. 


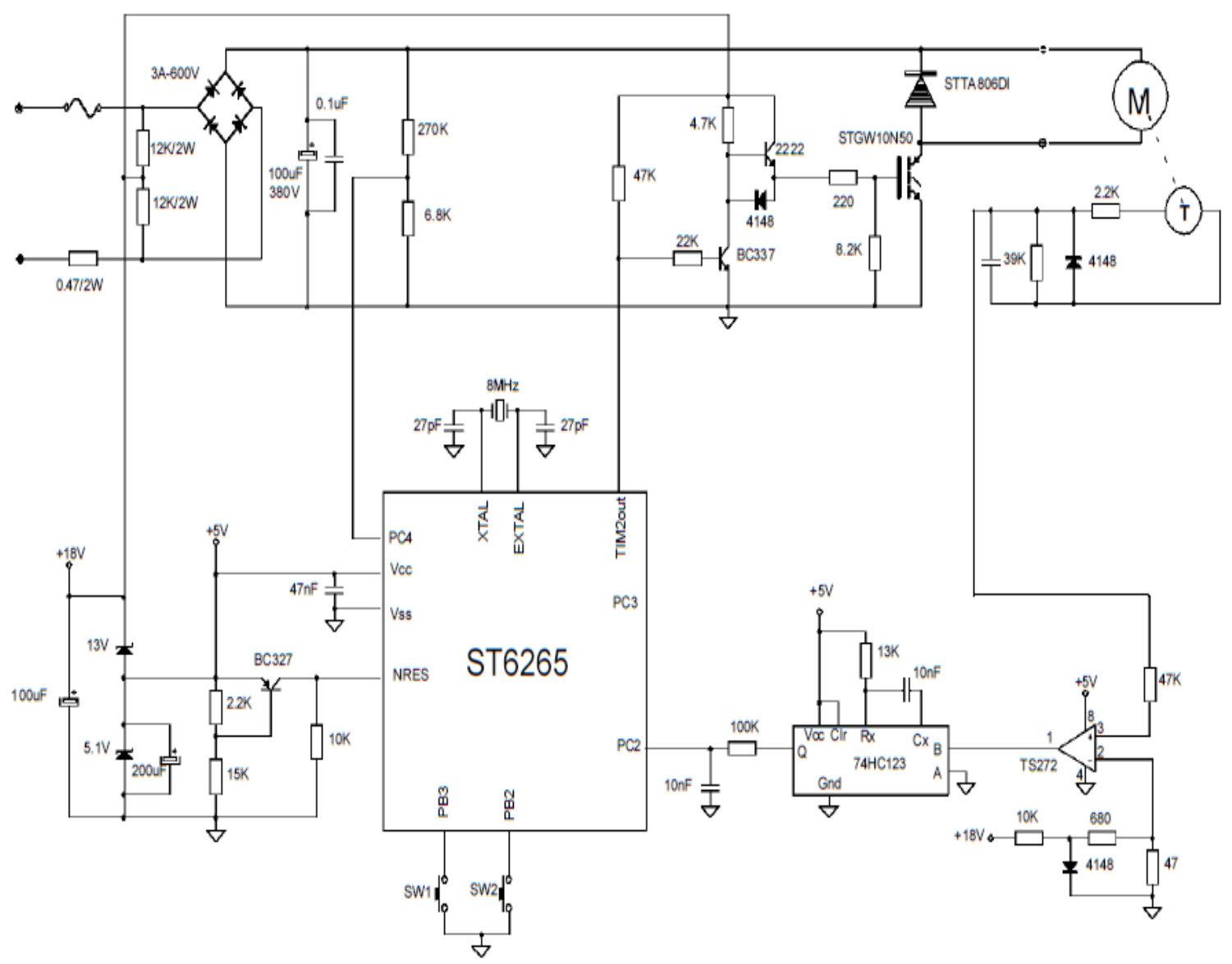

Figure 27: Complete circuit diagram of speed control of armature controlled DC motor using ST6265 (Fuzzy controlled MCU) and STA806DI (free wheeling diode)

\section{Authors}

Rahul Malhotra is currently pursuing Ph.D. degree from Punjab Technical University His area of interest includes Wireless Communication, Adhoc Networks, Fuzzy Logic \& Genetic Algorithms. He is a member of Indian Society for Technical Education, The Institution of Electronics \& Telecommunication Engineers (India), Institution of Engineers (India)

Yaduvir Singh obtained Bachelor of Engineering from Dayalbagh Educational Institute (Deemed University, Agra) in Year 1991, Master of Engineering in Control \& Instrumentation Engineering from Motilal Nehru National Institute of Technology, University of Allahabad in Year 1993, Master of Business Management from Newport University, USA in year 1997 and Doctor of Philosophy from Thapar University, Patiala in year 2004. He joined Thapar University as Assistant Professor in 2000. He is currently working as Associate Professor in the University.

Narinder Singh has received his Bachelor of Engineering from Thapar Institute of Engineering and Technology, Patiala, India under Punjabi University in year 1964 and M. Tech degree from Indian Institute of Technology, Roorkee in 1968 and Ph.D degree from Indian Institute of Engineering, Roorkee in 1987. He served as a Professor in Thapar University, Patiala. Later, he joined Giani Zail Singh College of Engineering \& Technology, Bathinda. He is currently working as Director in GGS College of Engineering \& Technology,Talwandi sabo,Bathinda. His field of interest includes Surveying, Fuzzy Logic, and Genetic Algorithm optimization. 